\section{EFFICACY AND TOXICITY OF SINGLE AGENT IMMUNE CHECKPOINT INHIBITORS AMONG ADULTS WITH CANCER AGED $\geq 80$ YEARS: A MULTICENTER INTERNATIONAL COHORT STUDY}

${ }^{1}$ Caroline Nebhan*, ${ }^{2}$ Alessio Cortellini, ${ }^{3}$ Weije Ma, ${ }^{4}$ Teja Ganta, ${ }^{1}$ Haocan Song, ${ }^{1}$ Fei Ye ${ }^{1}$ Rebecca Irlmeier, ${ }^{4}$ Neha Debnath, ${ }^{5}$ Anwaar Saeed, ${ }^{5}$ Maluki Radford, ${ }^{6}$ Asrar Alahmadi, ${ }^{6}$ Akiva Diamond, ${ }^{7}$ Christopher Hoimes, ${ }^{6}$ Nikhil Ramaiya, ${ }^{8}$ Carolyn Presley, ${ }^{8}$ Dwight Owen, ${ }^{9}$ Sarah Abou Alaiwi, ${ }^{9}$ Amin Nassar, ${ }^{9}$ Biagio Ricciuti, ${ }^{10}$ Giuseppe Lamberti, ${ }^{11}$ Melissa Bersanelli, ${ }^{11}$ Chiara Casartelli, ${ }^{11}$ Sebastiano Buti, ${ }^{12}$ Paolo Marchetti, ${ }^{12}$ Raffaele Giusti, ${ }^{1}$ Marco Filetti, ${ }^{13}$ Vito Vanella, ${ }^{13}$ Domenico Mallardo, ${ }^{14}$ Shravanti Macherla, ${ }^{15}$ Tamara Sussman, ${ }^{16}$ Andrea Botticelli, ${ }^{17}$ Domenico Galetta, ${ }^{17}$ Annamaria Catino, ${ }^{17}$ Pamela Pizzutilo, ${ }^{18}$ Carlo Genova, ${ }^{19}$ Maria Giovanna Dal Bello, ${ }^{20}$ Foteini Kalofonou, ${ }^{20}$ Ella Daniels, ${ }^{13}$ Paolo Ascierto, ${ }^{20}$ David Pinato, ${ }^{9}$ Toni Choueiri, ${ }^{1}$ Douglas Johnson, ${ }^{4}$ Thomas Marron, ${ }^{3}$ Yinghong Wang, ${ }^{21}$ Abdul Rafeh Naqash. ${ }^{1}$ Vanderbilt University Medical Center, Nashville, TN, USA; ${ }^{2}$ University of L'Aquila, L'Aquila, Italy; ${ }^{3} \mathrm{MD}$ Anderson Cancer, Houston, TX, USA; ${ }^{4}$ cahn School of Medicine at Mount Sinai, New York, USA; ${ }^{5}$ University of Kansas Cancer Center, Kansas City, KS, USA; ${ }^{6}$ Case Western Reserve University, Cleveland, USA; ${ }^{7}$ Duke Cancer Institute, Durham, OH, USA; ${ }^{8}$ The Ohio State University Comprehensive, Columbus, OH, USA; ${ }^{9}$ Dana-Farber Cancer Center, Boston, MA, USA; ${ }^{10}$ University of Bologna, Bologna, Italy; ${ }^{11}$ University of Parma, Parma, Italy; ${ }^{12}$ Universitaria Sant'Andrea, Rome, Italy; ${ }^{13}$ Istituto Nazionale Tumori IRCCS, Napoli, Italy; ${ }^{14}$ East Carolina University Brody School of, Greenville, USA; ${ }^{15}$ Cleveland Clinic Foundation, Cleveland, OH, USA; ${ }^{16}$ Sapienza University of Rome, Rome, Italy; ${ }^{17}$ IRCCS Istituto Tumori Giovanni Paolo II, Bari, Italy; ${ }^{18}$ Università degli Studi di Genova, Genova, Italy; ${ }^{19}$ IRCCS Ospedale Policlinico San Martino, Genova, Italy; ${ }^{20}$ Imperial College London, London, UK; ${ }^{21}$ National Cancer Institute, Silver Spring, MD, USA

Background Immune checkpoint inhibitors (ICIs) are approved by the U.S. Food\&Drug Administration in over 17 tumor types. Older adult patients make up about a quarter of all cancer patients but are historically understudied in cancer clinical trials. ICIs are associated with immune-related adverse events (irAEs), which may be particularly morbid for older adult patients with underlying comorbidities and impaired functional status. In this study, we provide insight into the real-world safety and efficacy of ICIs among older adult patients ( $\geq 80$ years) with cancer.

Methods This is a multicenter, international retrospective study of tumor-agnostic older adult patients with cancer treated with single-agent ICIs between 2010-2019 from 18 academic centers in the U.S. and Europe. A cohort of 928 patients aged $\geq 80$ years during treatment with ICI was assembled and analyzed to evaluate clinical outcomes and irAE patterns in older adult patients treated with single-agent ICIs.

Results Median age at ICI initiation was 83.0 years (range 75.8-97.0). Most patients (86.9\%) were treated with anti-PD1 therapy. Among the full cohort, the three most common tumors were non-small cell lung cancer (NSCLC, 37.2\%, $\mathrm{n}=345)$, melanoma $(35.5 \%, \mathrm{n}=329)$, and genitourinary (GU) tumors $(16.5 \%, \mathrm{n}=153)$. Objective response rates for patients with NSCLC, melanoma, and GU tumors were $32.2 \%$, $39.3 \%$, and 26.2\%, respectively. Median progression-free survival (PFS) was 6.7 months (95\%CI, 5.2-8.6) for patients with NSCLC, 11.1 months (95\%CI, 8.9-16.0) for patients with melanoma, and 6.0 months $(95 \%$ CI, 5.0-10.7) for patients with GU malignancy. Median overall survival (OS) was 10.9 months $(95 \% \mathrm{CI}, 8.6-13.1)$ for patients with NSCLC, 30.0 months (95\%CI, 23.6-46.4) for patients with melanoma, and 15.0 months $(95 \% \mathrm{CI}$ 9.1-25.4) for GU patients (Figure 1A-C). Within histology-specific cohorts (NSCLC, melanoma and GU), clinical outcomes were similar across age subgroups $(<85,85-89,>90)$. Among all patients $(\mathrm{N}=928), 41.3 \%$ experienced $\geq 1 \mathrm{irAE}(\mathrm{s})$, including $12.2 \%$ reported to be grade (G)3-4. No irAE-related deaths occurred. The median time to irAE onset was 9.8 weeks; $57 \%$ occurred within the first 3 months after ICI initiation. ICI was discontinued due to irAEs in $16.1 \%$ patients. There was no significant difference in the rate of irAEs among patients age $<85$, $85-89$, and $\geq 90$ years $(p=0.15)$. Despite similar rates of $G 3+$ irAEs, ICIs were discontinued due to irAE more than twice as often among patients $\geq 90$ years compared to patients $<90$ years $(30.9 \%$ vs. $15.1 \%, \mathrm{p}=0.008)$ (table 1$)$.

Conclusions ICIs are effective and generally well-tolerated among older patients with cancer. However, ICI discontinuation due to irAE is more frequent with increasing age.

http://dx.doi.org/10.1136/jitc-2021-SITC2021.239 